\title{
Dynamic Shelter Structure
}

\author{
F. Maden \& D. Ölmez \\ Department of Architecture, Yaşar University, İzmir, Turkey \\ Ş. Gür \& M.Y. Uncu \\ Department of Architecture, Izmir Institute of Technology, İzmir, Turkey \\ C. Mitropoulou \\ ACE Hellas, Athens, Greece
}

\begin{abstract}
Dynamic building envelopes have entered the mainstream practice of architecture in the last decades. Such dynamic systems are capable of changing their geometric configurations repeatedly and reversibly relative to environmental conditions and occupant requirements. Thus, they may offer innovative building solutions by folding, expanding or curling. This study proposes a dynamic shelter structure that provides several shape options in response to the changing needs. In order to generate the shelter structure, first, loop assembly method used for developing the structure is introduced. Then, a parametric model is built in Grasshopper ${ }^{\circledR}$ not only to analyze the geometric properties of the loops and their alternative geometric forms but also to develop a flexible tool allowing changes at topological, geometrical and structural levels. Based on the geometric analysis, the structural mechanism is constructed. Transformation capability and possible configurations are studied. The proposed structure can transform itself into multiple forms (from planar con-figuration to S-shaped and reversed S-shaped configurations) with single DOF although the existing single DOF scissor structures can deploy between two geometric shapes.
\end{abstract}

\section{INTRODUCTION}

Adapting to different climates and landscapes, being able to take many forms depending on the requirements of users and their capacity to meet rapidly changing circumstances are what makes transformable/deployable structures so promising. A deployable structure incorporating a mechanism has at least one kinematic degree-of-freedom (DOF). This gives the structure the ability to change configuration, such as transforming from compact to expanded forms. Most of the time, this is a reversible action and they perform their architectural function only in the fully deployed configuration. Various structural systems in a wide range have been proposed as deployable/transformable systems such as scissor (or pantographic) structures, deployable tensegrity, structural origami, foldable membrane structures and -more recently- tensairity. The proposed dynamic shelter structure in this paper belongs to the structural group with rigid links composed of scissor elements. It has the ability to change its geometric shape into different forms with single DOF. The novelty of the structure is its generation method and varying forms. Rather than using the current design approach in the literature, another approach called loop assembly method is used to generate the structure that is composed of kite loops.

\section{GEOMETRIC DESIGN METHOD}

Loop assembly method is based on constructing the scissor structures by using different loop types. Kite loop is used to construct the dynamic shelter structure. First, identical kite loops are assembled on a plane and then scissor element is drawn on the edges of the two adjacent kite loops. The scissor element is angulated-shaped which lies on the long side of the first loop and 
the short side of the second loop. The kink angle of the angulated element defines the transformation behavior of the loop assembly. Using the kite loops, two different loop assemblies are generated which are arch-shaped loop assembly and S-shaped loop assembly. Both types allows generating three different geometric configurations with single DOF.

\section{THE DYNAMIC SHELTER STRUCTURE}

Covering approximately $140 \mathrm{~m}^{2}$, the proposed structure is implemented as a dynamic shelter that can serve for different temporary or permanent activities. To create the dynamic shelter structure, aforementioned S-shaped loop assembly is used. The structure is composed of eleven S-shaped modules that are connected to each other by X-shaped elements. Each S-shaped module is composed of nine kite loops. The kite loops comprise of angulated elements (PropLongArm $=70 \mathrm{~cm}$ and PropShortArm $=35 \mathrm{~cm}$ ) that are hinged at their mid- and end- points. In order to convert the created structural mechanism into a load bearing structure, telescopic beam elements are added to the system, which allow linear translation of the system in horizontal direction. Those elements are supported by bracing elements and connected to $7 \mathrm{~m}$-high columns by pin connections. As the telescopic beam elements extend or shorten, the curvature of the structure changes. There are revolute joints at the connection points of the loops to the beams and columns, which provide the required rotational motion. By this means, the structure changes its geometry from the S-shape to the reversed S-shape. In addition to these forms, the structure can also remain at planar configuration that allows generating a flat surface.

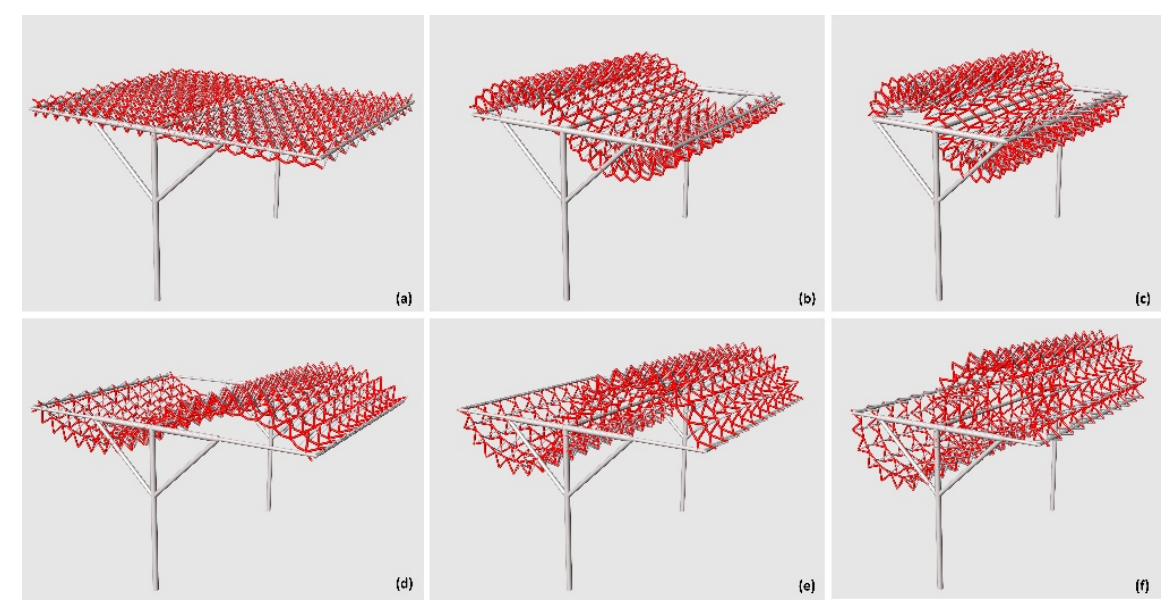

Figure 1. Transformation process of dynamic shelter structure

\section{CONCLUSION}

In this paper, a dynamic shelter structure has been presented which has the ability to transform itself into different geometric shapes. The structure has been created using the loop assembly method that allows more flexibility on form transformation with single DOF even though it is required to use more DOF in the existing examples to generate different geometries. This reveals the superiority of the proposed structure over the existing ones since the dynamic shelter structure not only transforms from S-shaped geometry to the reversed S-shaped form, but also provides a planar configuration. According to the changing circumstances, the structure responds to the user needs by geometric transformations.

\section{ACKNOWLEDGMENT}

This work is a part of OptArch project that has received funding from the European Union's Horizon 2020 Research and Innovation programme under the Marie Skłodowska-Curie grant agreement No 689983. 